\title{
Genetic Algorithm Based Control System Design of a Self-Excited Induction Generator
}

\author{
A.-F. Attia, H. Soliman, M. Sabry
}

This paper presents an application of the genetic algorithm (GA) for optimizing controller gains of the Self-Excited Induction Generator (SEIG) driven by the Wind Energy Conversion Scheme (WECS). The proposed genetic algorithm is introduced to adapt the integral gains of the conventional controllers of the active and reactive control loop of the system under study, where GA calculates the optimum value for the gains of the variables based on the best dynamic performance and a domain search of the integral gains. The proposed genetic algorithm is used to regulate the terminal voltage or reactive power control, by adjusting the self excitation, and to control the mechanical input power or active power control by adapting the blade angle of WECS, in order to adjust the stator frequency. The GA is used for optimizing these gains, for an active and reactive power loop, by solving the related optimization problem. The simulation results show a better dynamic performance using the GA than using the conventional PI controller for active and reactive control.

Keywords: genetic algorithms, conventional controllers, self-excited induction generator.

\section{Introduction}

Studies of self-excited induction generators have been investigated since 1935. Many papers dealing with various problems in the field of SEIG have been published. The primary advantages of SEIG are lower maintenance costs, better transient performance, lack of a dc power supply for field excitation, brushless construction (squirrel-cage rotor), etc. In addition, induction generators have been widely employed to operate as wind-turbine generators and small hydroelectric generators of isolated power systems. Induction generators can also be connected to large power systems, to inject electric power $[1,2]$.

The generator action takes place, when the rotor speed of the induction generator is greater than the synchronous speed of the air-gap-revolving field. Various configurations for connecting SEIG to a large power system have been discussed in many publications. This research concentrates on the dynamic performance of an isolated SEIG, driven by wind energy, to supply an isolated static load. A D-Q axis equivalent circuit model based on various reference frames, extracted from fundamental machine theory, is created to study SEIG performance in dynamic case [2]. This paper studies SEIG performance, when equipped with a switching capacitor bank, using a controller based on GAs to adjust the duty cycle and adjusting the stator frequency via the pitch control.

Genetic algorithms are search algorithms that simulate the process of natural selection and survival of the fittest [3]. The GA starts off with a population of randomly generated chromosomes, and advances toward better chromosomes in a sequence of generations. During each generation, the fitness of each solution is evaluated and solutions are selected for reproduction based on their fitness. Then, the chromosomes with higher fitness have higher probabilities of having more copies in the following generation, while the chromosomes with worst fitness are eliminated. Then a roulette wheel cheme is applied for reproduction. Consequently, the new population of chromosomes is formed using a selection mechanism and specific genetic operators such as crossover and mutation. Recently, genetic algorithms have received considerable attention. Global optimization utilizes techniques that can distinguish between the global optimum and numerous local optima within a region of interest. Many papers have been published using GA to optimize PI and PID controllers [4].

In this paper, the mathematical model of SEIG driven by WECS is simulated using the MATLAB/SIMULINK package to solve its differential equations. Two controllers have been developed for the system under study. The first of these is the reactive controller to adjust the terminal voltage at the rated value, by controlling the duty cycle of the switching capacitor bank. The second controller is the active controller, which adjusts the input mechanical power to the generator and thus keeps the stator frequency constant. This is achieved by controlling the pitch angle of the blade of the wind turbine.

Both controllers are implemented using the conventional PI controller. Then, the integral gains of both PI controllers are optimized using the GA technique. Fig. 1 shows the block diagram for the system under study. It consists of a self-excited induction generator driven by a wind energy conversion scheme connected to an isolated load. In addition, the system under study is equipped with the reactive and active controller's loops, using a PI controller. Then, the integral gains are tuned using the GA algorithm.

\section{Mathematical model for SEIG driven by WECS}

\subsection{Electrical equations for SEIG}

Fig. 2 shows the $d-q$ axis equivalent-circuit model for a no-load, three-phase symmetrical induction generator. The stator and rotor voltage equations using Krause transformation [1, 2], based on a stationary reference frame, are given in Appendix A. 


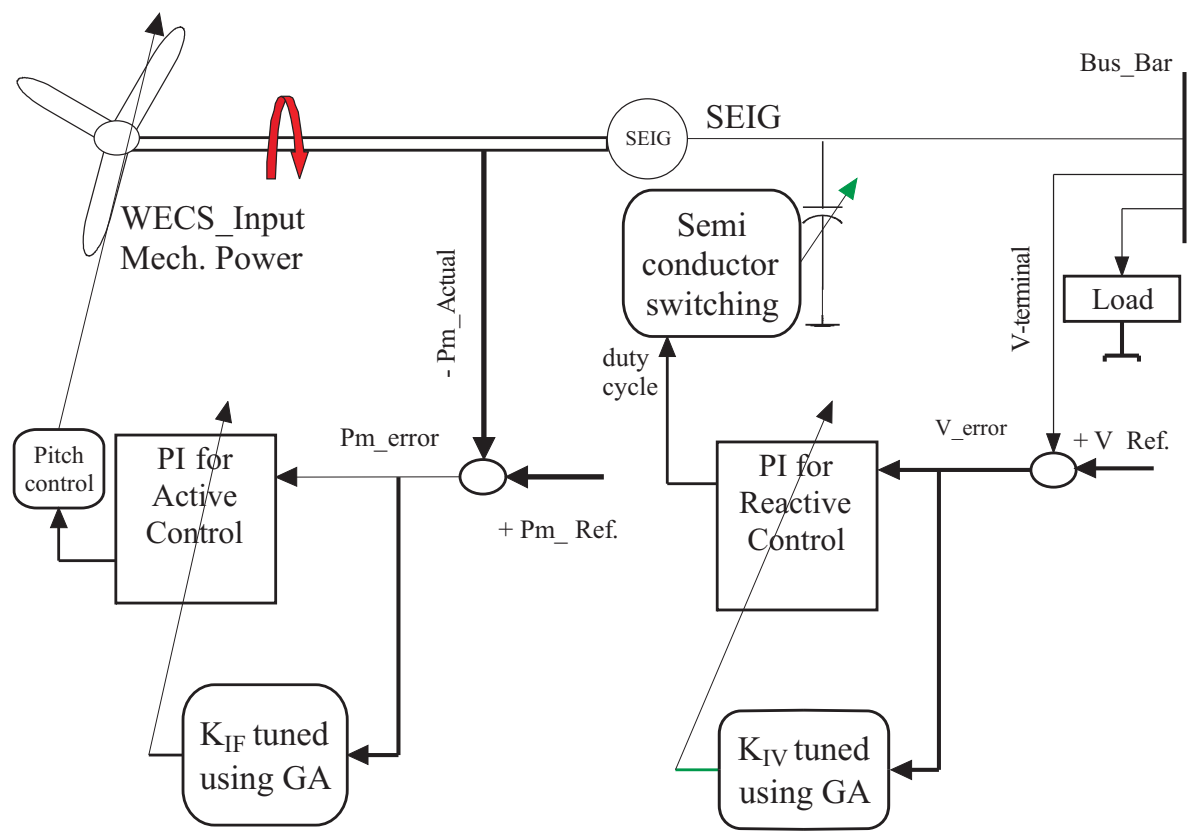

Fig. 1: System under study

\subsection{Mechanical equations for WECS}

The mechanical equations relating the power coefficient of the wind turbine, tip speed ratio $\mu$ and pitch angle $\beta$ are given in Appendix A [5]. The analysis of SEIG in this paper is based on the following assumptions [1]:

- All parameters of the machine can be considered constant except $X_{m}$

- Per-unit values of both stator and rotor leakage reactance are equal

- Core loss in the excitation branch is neglected

- Space and time harmonic effects are ignored.

\subsection{Equivalent circuit}

The $d-q$ axsis equivalent-circuit models for a no-load, three-phase symmetrical induction generator are shown in

Fig. 2a and Fig. 2b. The equivalent-circuit parameters shown in these figures are based on the machine data in Appendix B $[1,2]$.

The equation of motion of the rotating part of the combined studied SEIG and the wind turbine is also included in the system in order to provide a detailed simulation model.

\subsection{Reactive control and switching capacitor bank technique}

\subsubsection{The switching capacitor bank}

Capacitor switching has been discarded in the past because of the practical difficulties involved [6], i.e. the occurrence of voltage and current transients. It has been argued, and justly so, that current 'spikes', for example, would inevitably exceed the maximum current rating as well as the $(\mathrm{d} i / \mathrm{d} t)$

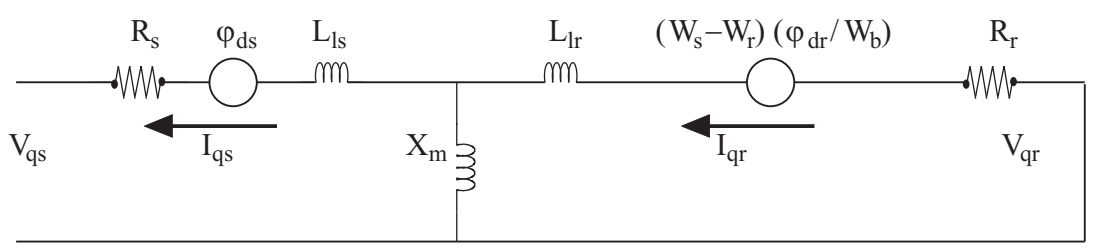

a)

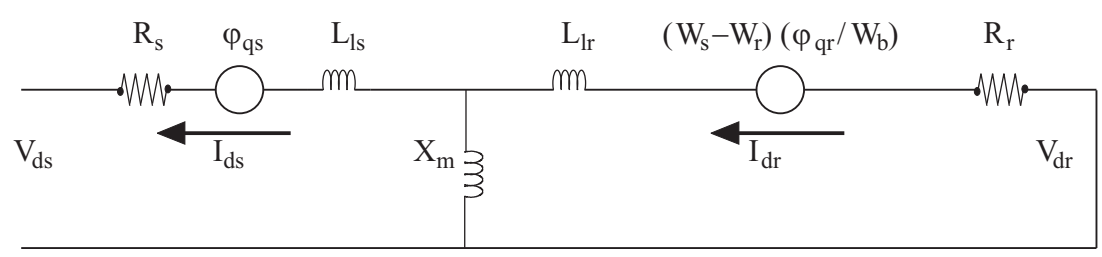

b)

Fig. 2: a) Equivalent circuit of an induction generator for quadrate axis, b) Equivalent circuit of an induction generator for direct axis 


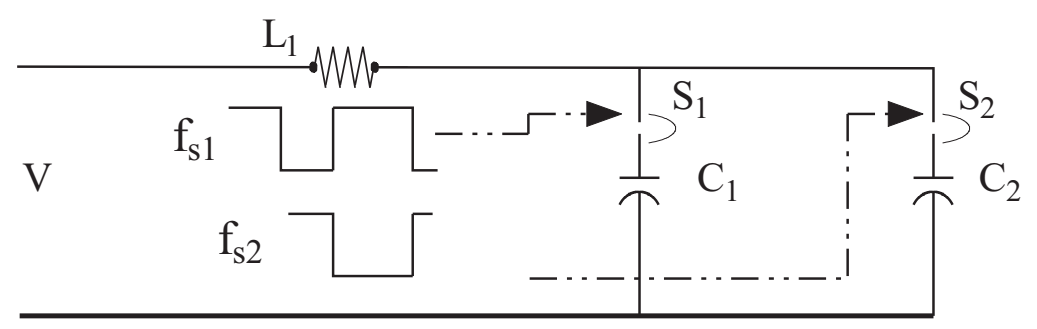

Fig 3: Semi conductor switches $\left(\mathrm{S}_{1}, \mathrm{~S}_{2}\right)$ circuit for capacitor bank

value of a particular semiconductor switch. The only way out of this dilemma would be to design the semiconductor switch to withstand the transient value at the switching instant.

An equivalent circuit of the switching capacitor bank with a controlled value of the duty cycle is shown in Fig. 3. In this figure, the switches are operated in anti-phase, i.e. the switching function $f_{s 2}$ which controls switch $\mathrm{S}_{2}$ is the inverse function of $f_{s 1}$ which controls switch $\mathrm{S}_{1}$. In other words, switch $\mathrm{S}_{2}$ is closed during the time when switch $S_{1}$ is open, and vice versa. This means that $S_{1}$ and $S_{2}$ of branch 1 and 2 are operated in such a manner that one switch is closed while the other is open.

\subsubsection{Reactive control through the switching capacitor bank technique}

In the system under study given in Fig. 1, the controller input is the voltage error for the reactive power controller, and the output of the controller performs the value of the duty cycle $\lambda$. The duty cycle is used as an input to the semiconductor switches to adjust the capacitor bank $C_{\text {eff }}$ value according to the need for the effective value of the excitation, which regulates the terminal voltage. Accordingly the semiconductor switching technique as explained in the above section and, hence, the terminal voltage is controlled by adjusting the self-excitation through automatic switching of the capacitor bank.

\subsection{The active power control}

Active control is applied to the system under study by adjusting the pitch angle of the wind turbine blades. This is used to keep the SEIG operating at a constant stator frequency and to avoid the effect of the disturbance. The pitch angle is a function of the power coefficient $C_{p}$ of the wind turbine WECS. The value of $C_{p}$ is calculated using the pitch angle according to Eq. (14), given in Appendix A. Consequently, the best adjustment for the value of the pitch angle improves the mechanical power regulation, which achieves better adaptation for the frequency of all systems. Accordingly, the active power control regulates the mechanical power of the wind turbine.

\section{Proportional plus integral (PI) controllers}

First, the PI controller using a fixed gain is applied to the system under study. Then, the integral gain $K_{I}$ of the PI controller is varied linearly with reference to terminal voltage error $e_{V}$, while proportional gain $K_{P}$ is fixed. The voltage or frequency error is used as an input variable to the PI control-

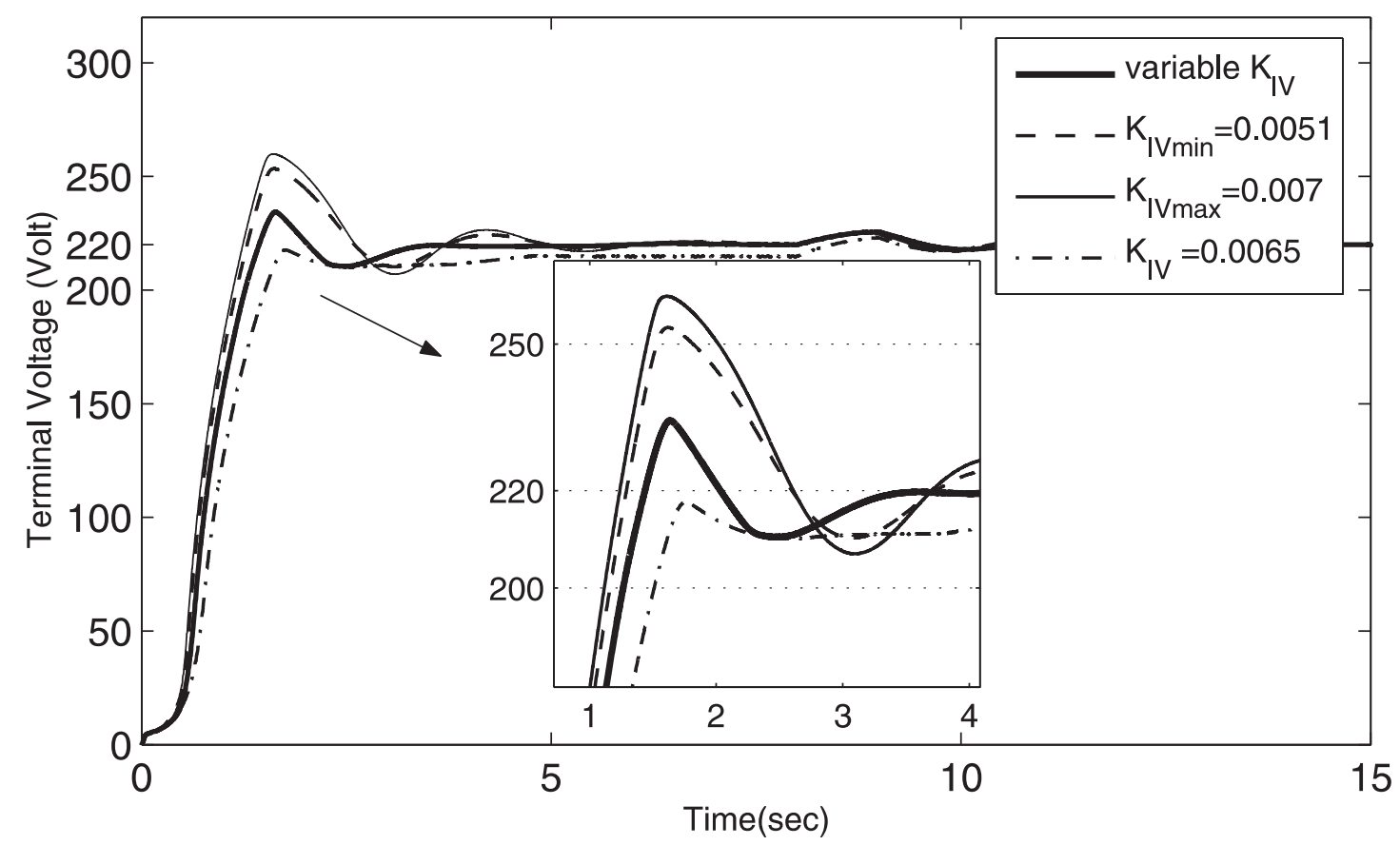

Fig. 4: Dynamic response of the terminal voltage with different values of integral gain for reactive control 


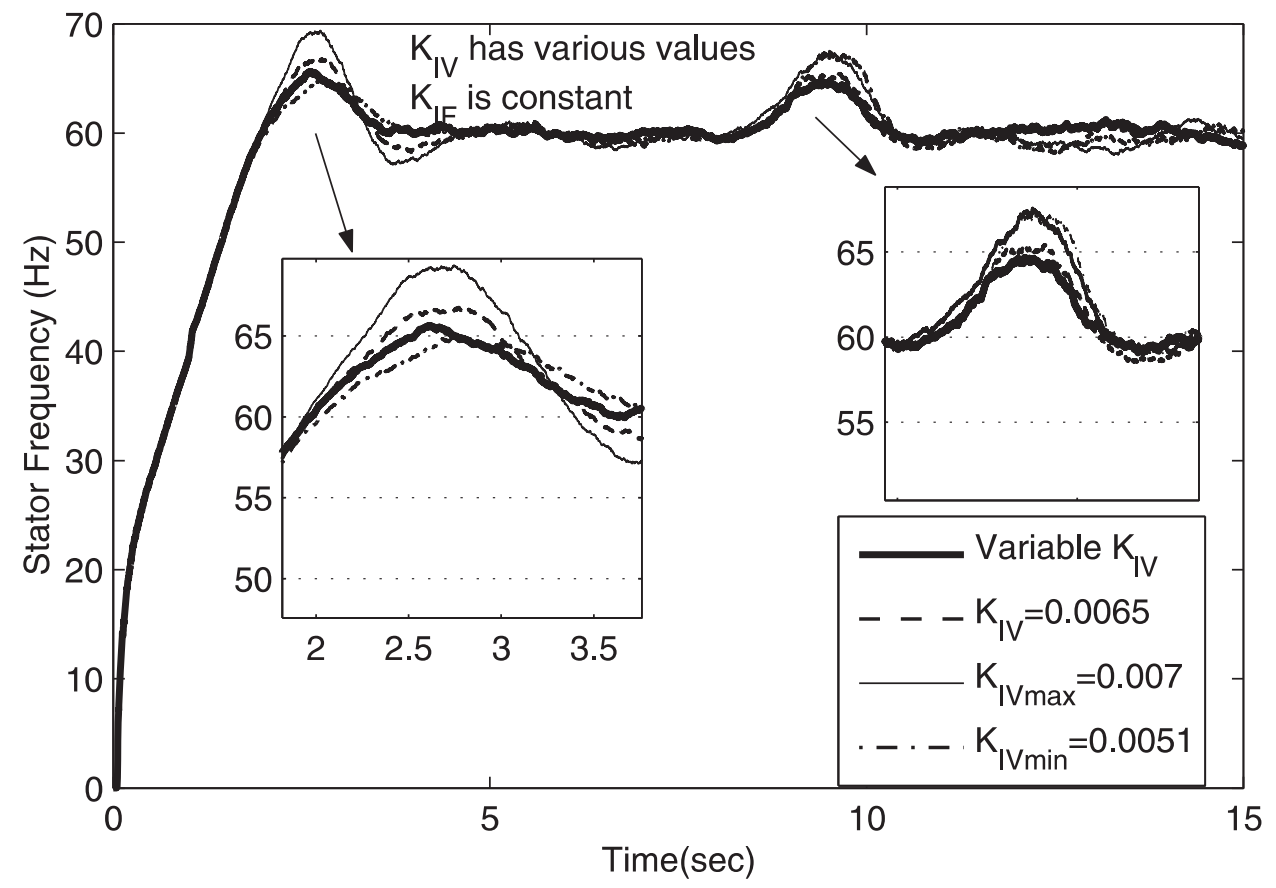

Fig. 5: Dynamic response of the stator frequency

ler, then the output is used to regulate the duty cycle of the switching capacitor bank in the reactive controller, while the output of the active controller the output is utilized to tune up the pitch angle of the wind turbine to adjust the system frequency.

Fig. 4 shows the simulation results for the system under study when starting against a step change in the reference voltage. Then the system is subjected to a sudden change in the local electric load. This simulation result is carried out for different values of fixed integral gain. Figs. 5, 6 and 7 show the simulation results for the stator frequency, the duty cycle and the stator current for the previous condition indicated in Fig. 4.

These simulation results show that the dynamic performance is changed, as regards percentage over shoot (p.o.s), rising time and oscillation, by changing the value of the integral gain. Thus, the idea of driving the system using a variable integral gain is introduced to achieve the benefits of using high and small integral gains, as follows.

From Fig. 4, a higher value of the integral gain, $K_{I V}=0.007$, is associated with a shorter rising time but the

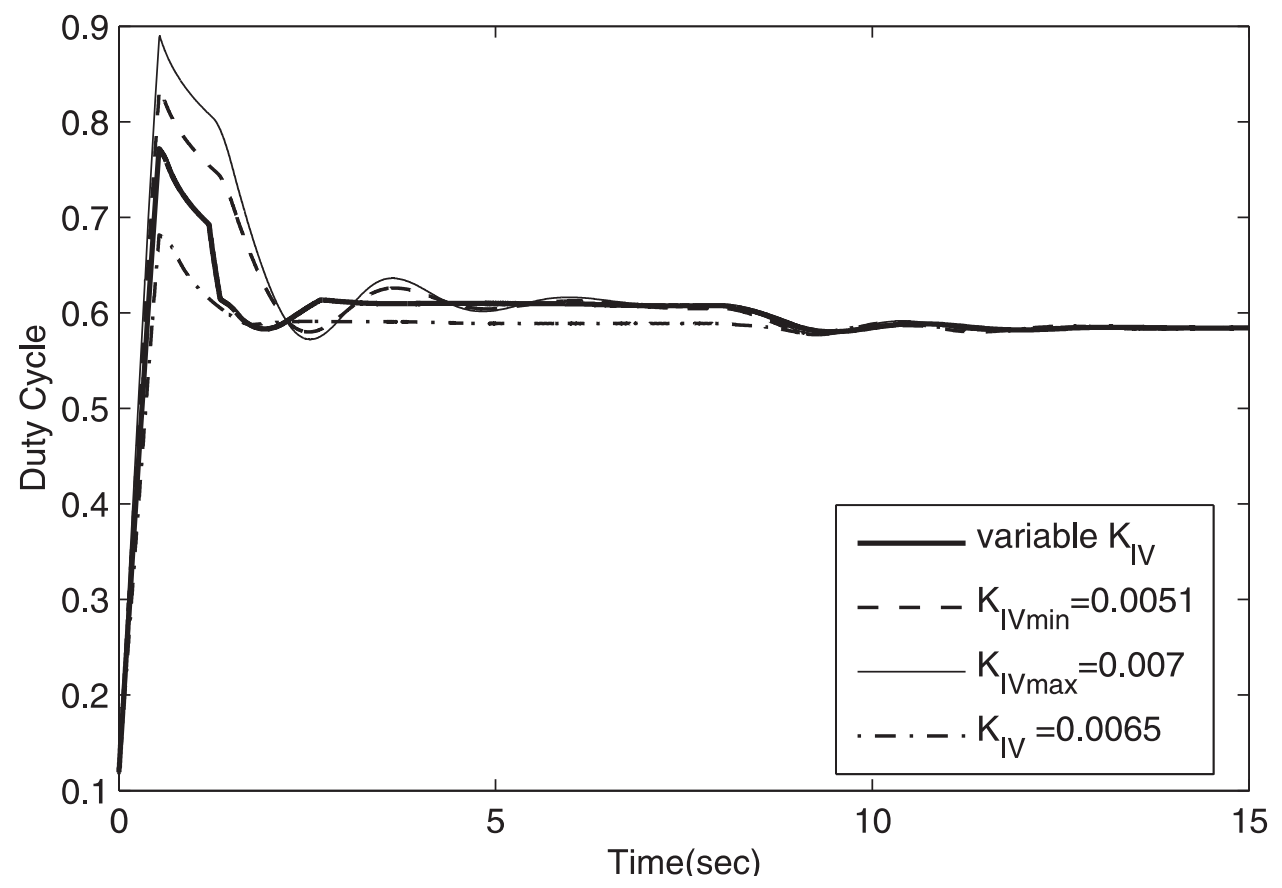

Fig. 6: Dynamic response of the duty cycle 


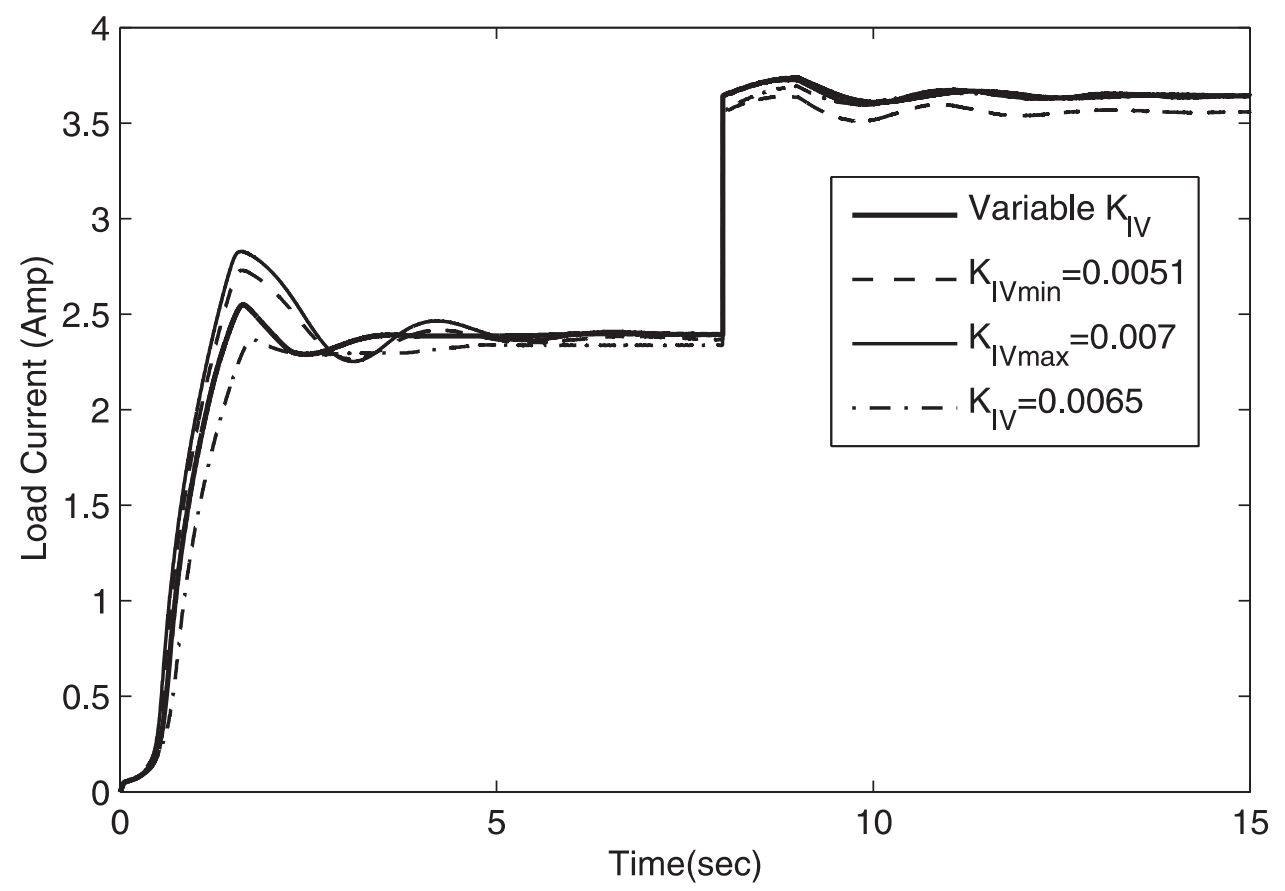

Fig. 7: Dynamic response of the load current

p.o.s and oscillation are greater than the other values of $K_{I V}$. Meanwhile, the lowest value of $K_{I V}=0.0051$ is associated with negligible p.o.s and oscillation and the rising time is greater than other values of $K_{I V}$. Fig. 8 show the variation of the integral gain during the starting period versus time.

\subsection{PI-Controller with a variable gain}

A program has been developed to compute the value of the variable integral gain $K_{I V}$ using the following rule:

$$
\begin{aligned}
& \text { if }\left(e_{V}<e_{V \min }\right) \text {, } \\
& K_{I V}=K_{I V \min } ; \\
& \text { elseif }\left(e_{V}>e_{V \max }\right), \\
& \quad K_{I V}=K_{I V \max } ; \\
& \text { else }\left(e_{V \min }<e_{V}<e_{V \max }\right), \\
& \quad M=\left(K_{I V \max }-K_{I V \min }\right) /\left(e_{V \max }-e_{V \min }\right) \text {; } \\
& \quad C=K_{I V \min }-M \times e_{V \min } ; \\
& \quad K_{I V}=\left(M \times e_{V}\right)+C ; \\
& \text { end }
\end{aligned}
$$

where $e_{V}$ is the voltage error, $e_{V \min }$ and $e_{V \max }$ are the minimum and maximum values of the voltage error, respectively, $K_{I V \min }$ and $K_{I V \max }$ are the minimum and maximum values of the variable integral gain, respectively, $C$ is a constant and $M$ is the slop constant of the linear part. Fig. 9 shows the above rule used to calculate the variable $K_{I V}$. The value of $e_{V \min }$ and $e_{V \max }$ is obtained by trial and error to give the best dynamic performance.

Figs. 4, 5, 6 and 7 also show the dynamic performance of the overall system when equipped with this variable integral gain compared with other fixed integral gains. The simulation results depict an improvement in the dynamic performance when using the variable KIV.

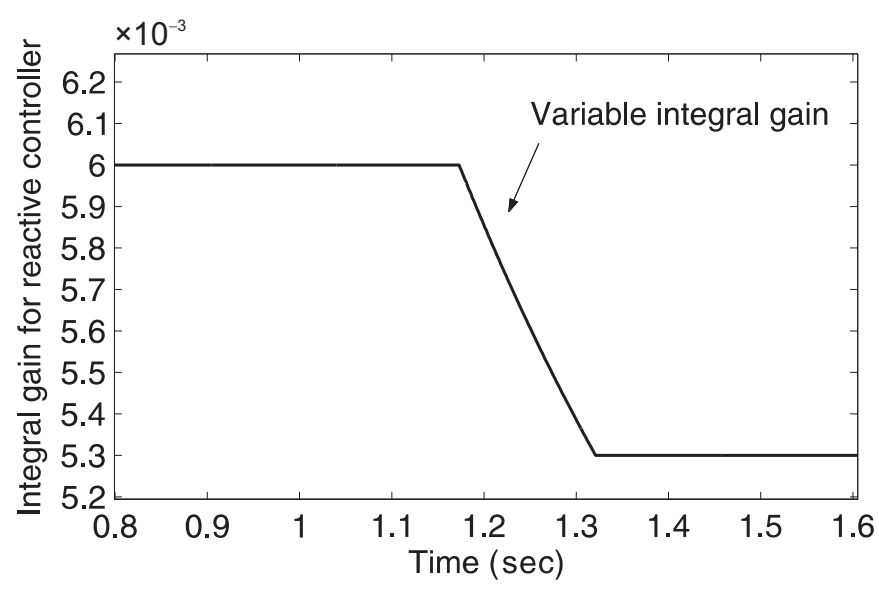

Fig. 8: Variable Integral gain for PI controller

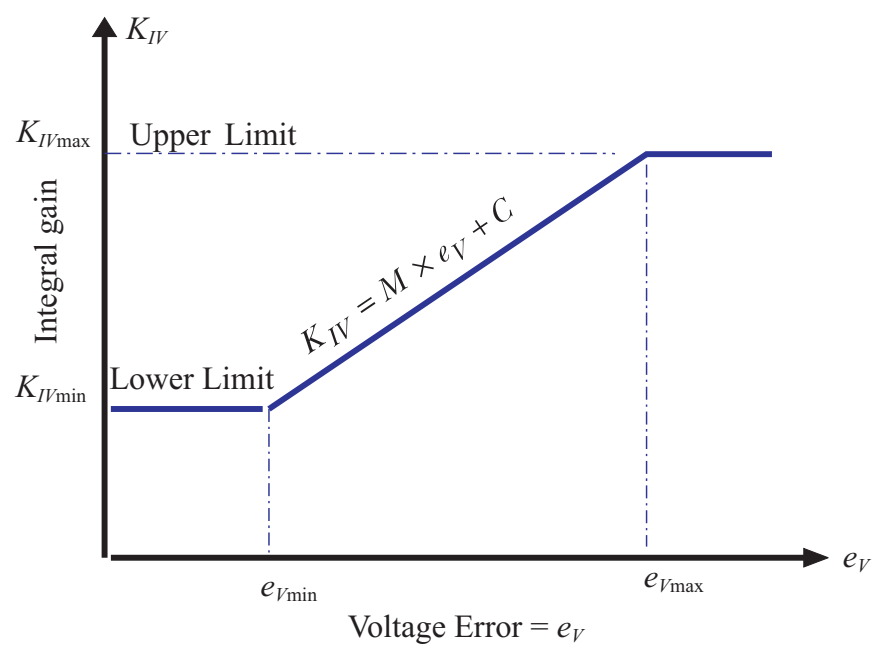

Fig. 9: Variable integral gain for a PI controller 


\section{Genetic algorithm with a constrained search space for optimizing PI controller gains}

The integral gain of the second PI controller is optimized based on the Genetic Algorithm. GA is used to calculate the optimum value of the variables based on the best dynamic performance and a domain search of the variable. The objective function used in the GA technique is $F=1 /(1+J)$, where $J$ is the minimum cost function, which will be defined later. GA uses its operators and functions to find the values of $K_{I V}$ and $K_{I F}$ of the PI controllers to achieve better dynamic performance of the overall system. These values of gains lead to the optimum value of gains for which the system achieves the desired values by improving the P. O. S, rising time and oscillations. The main aspects of the proposed GA approach for optimizing the gains of PI controllers, and the flowchart procedure for the GA optimization process, are shown in Fig. 10.

\subsection{Representation of PI controller gains}

The PI gains are formulated using the GA approach, where all the gains are represented in a chromosome. The chromosome representation determines the GA structure. The parameter gains are encoded in a chromosome. The PI controller gains are initially started using minimum values of the domain search for PI gains. Based on the simulation

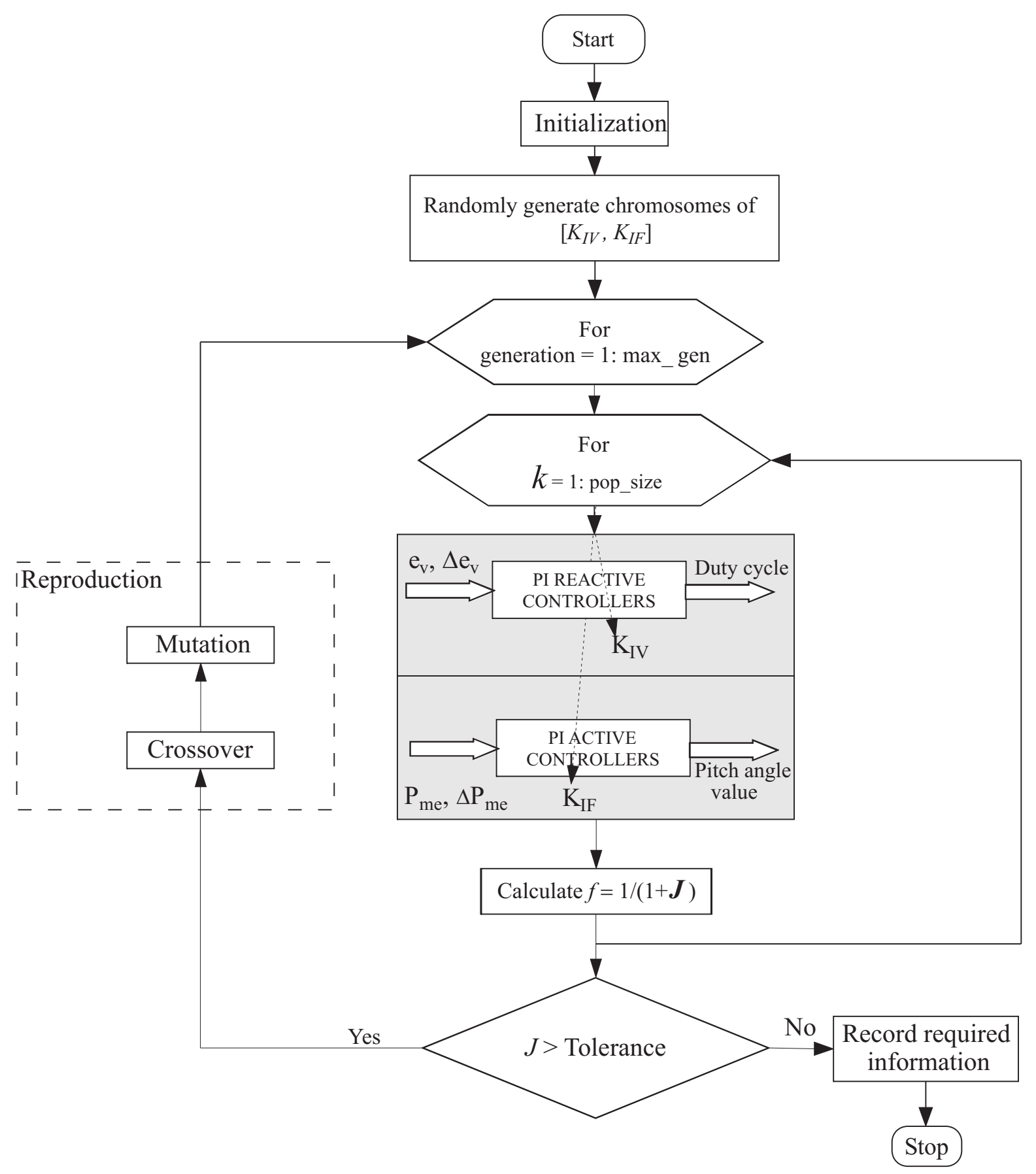

Fig. 10: Flowchart of GA approach for optimizing PIC gains 


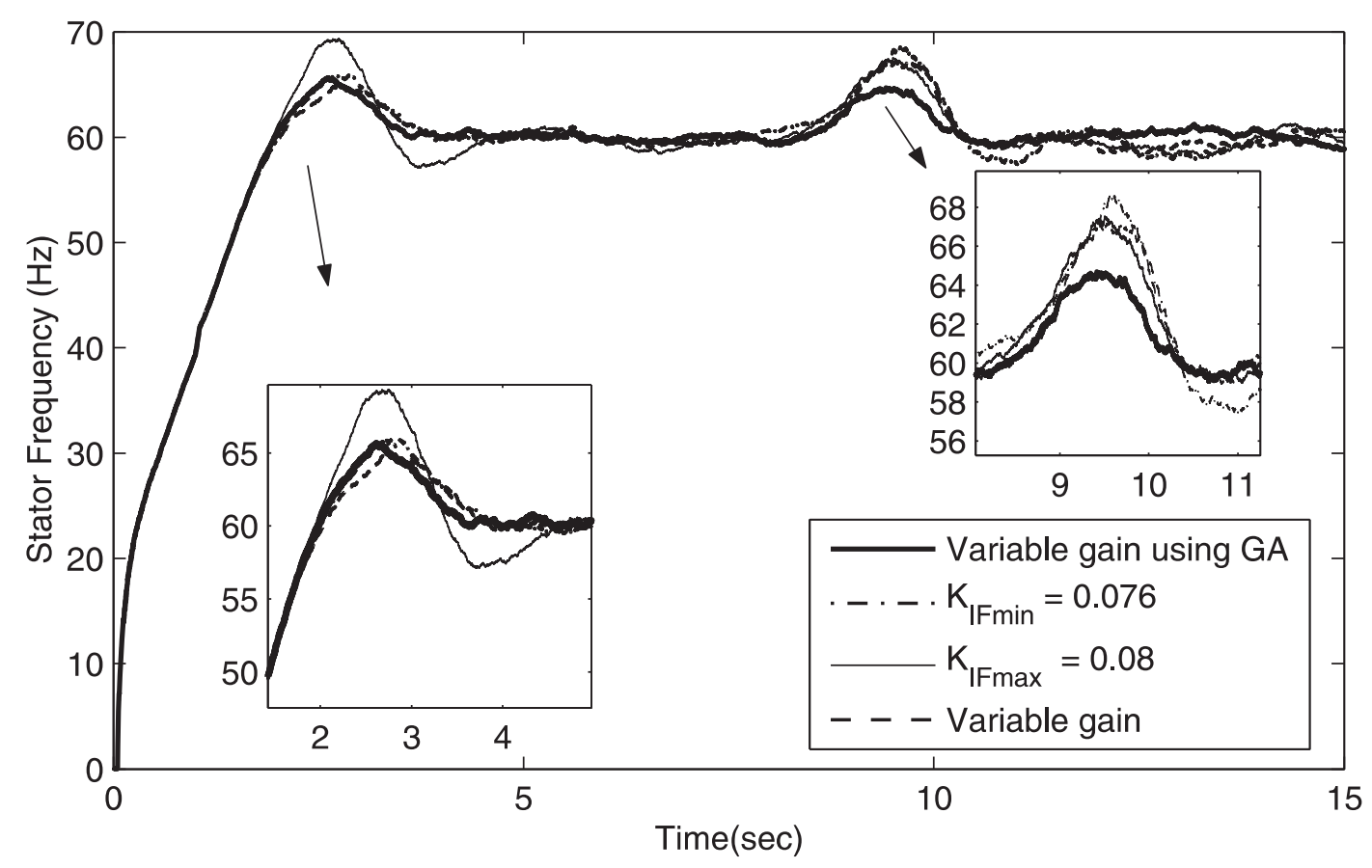

Fig. 11: Stator frequency for PI and PI \& GA controllers

results given in the previous sections, the values of $\left[K_{I V \max }\right.$, $\left.K_{I V \min }\right]$ are shown in Fig. 4 , while the values of $\left[K_{I F \max }\right.$, $\left.K_{I F \text { min }}\right]$ are shown in Fig. 11 . The acceptable domain search for each gain is defined as $\left[K_{I V \max }, K_{I V \min }\right]$ and $\left[K_{I F \max }\right.$, $\left.K_{I F \min }\right]$ based on five times less and five times more than the gains obtained using the Ziegler-Nichols rule to satisfy minimum cost function $J$, as given in the following equation [7]:

$J=\int_{0}^{T}\left(\alpha_{1}|e(t)|+\beta_{1}\left|e^{\prime}(t)\right|+\gamma_{1}\left|e^{\prime \prime}(t)\right|\right) \mathrm{d} t$,

where; $e(t)$ is equal to $e_{V}$ or $P_{m e} ; e_{V}$ is the voltage error used for the reactive power control and $P_{m e}$ is the mechanical power error used for active power control, as shown in Fig. 1. The parameters $\alpha_{1}, \beta_{1}$ and $\gamma_{1}$ are weighting coefficients.

\subsection{Coding of PI controller gains}

The coded parameters are arranged on the basis of their constraints, as shown in Fig. 12, to form a chromosome of the population. The binary representation, given in Fig. 12, is the coded form for parameters with chromosome length equal to the sum of the bits for all parameters. In binary coding, the relation between the bit length $L_{i}$ and the corresponding bit resolution $R_{i}$ is given in the following equation [8]:

$$
R_{i}=\frac{U B_{i}-L B_{i}}{2^{L_{i}}-1},
$$

where $U B_{i}$ and $L B_{i}$ are the upper and lower bounds of parameter $i$, respectively. In the present case study, we assume bit resolution $R=10^{5}$ for all parameters. Fig. 12 shows the coded parameters of the PI controller gains for reactive and active power controllers, respectively. The chromosome length used in this paper was 20 bits, where the bit length of $K_{I V}$ equal 10 bits and the bit length of $K_{I F}$ equals 10 bits.

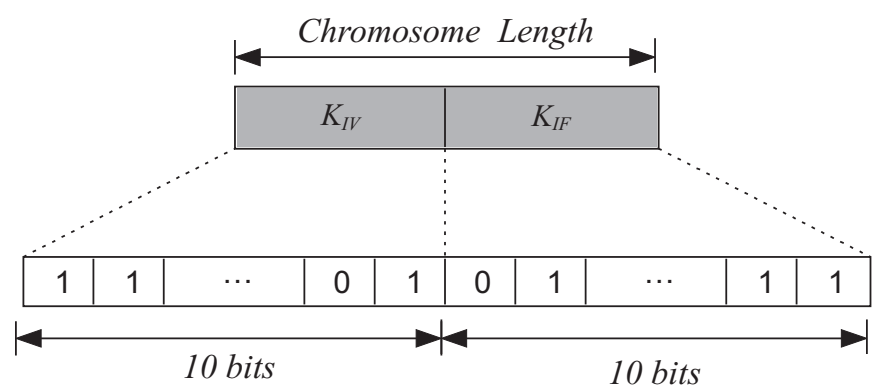

Fig. 12: Coded parameters of PI controller gains

\subsection{Selection function}

The selection strategy decides how to select individuals to be parents for new 'children'. The selection usually applies some selection pressure by favoring individuals with better fitness. After procreation, the suitable population consists, for example, of $L$ chromosomes, which are all initially randomized. Each chromosome has been evaluated and associated with fitness, and the current population undergoes the reproduction process to create the next population. Then, the "roulette wheel" selection scheme is used to determine the member of the new population. The roulette scheme is shown in Fig. 13. The chance on the roulette-wheel is adaptive and is given as $P_{\ell} / \sum P_{\ell}$ as in the Eq. (3) [8]:

$$
P_{\ell}=\frac{1}{J_{\ell}}, \quad \ell \in\{1, \ldots, L\},
$$

and $J_{\ell}$ is the performance of the model encoded in the chromosome measured in the terms used in Eq. (1). 


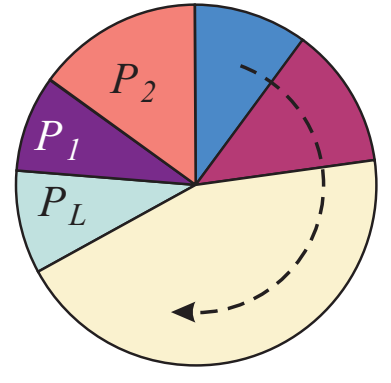

Fig. 13: Roulette wheel selection scheme

Maximizing the fitness function of each chromosome, which is inversely proportional to the performance criteria, Eq. (1) will damp the overshoot or the oscillations [8].

\subsection{Crossover and mutation operators}

The mating pool is formed, and crossover is applied. Then the mutation operation is applied, followed by the proposed GA approach. Finally, after these three operations, the overall fitness of the population is improved. The procedure is repeated until the termination condition is reached. The termination condition is the maximum allowable number of generations, or a certain value of $J$. This procedure is shown in the flowchart given in Fig. 10.

\section{Simulation results}

The nonlinear differential equation, which describes the system under study, was solved using the Runge-Kutta fifth order method, using the MATLAB Simulink package. The integration step value was automatically varied in this package. The relative tolerance was set at 0.00001 . The minimum and maximum step size was adjusted automatically. Several tests were carried out to validate the efficiency of the proposed control schemes.

The simulations show the comparison between the two proposed PI controllers. The system performance checks the terminal voltage $V_{L}$, the stator frequency $F_{s}$, the load current $I_{L}$ and the duty cycle versus time.

The overall system is tested against a sudden change in the load. This disturbance is made by applying a sudden change in the resistance part of the load impedance. The load is equivalent to the R-L series circuit with load resistance $R_{L}=80 \mathrm{ohm}$ and load inductance $L_{L}=0.12 \mathrm{H}$ per phase.

\section{PI controller based on GA}

\subsection{Dynamic performance due to sudden load variation}

Figs. 14, 15, 16 and 11 show the simulation results of the system under study using the PI controller based on GA for the terminal voltage, the stator current, the duty cycle and the stator frequency, respectively.

The simulation results show that the performance of a PI controller based on GA is much better, as regards maximum overshoot and rising time, than a PI controller with fixed or variable KIV. The simulation results show the effectiveness of the proposed controller, as shown in the previous figures.

\subsection{Simulation results due to sudden wind speed variation}

Other simulation results are obtained when the overall system is subjected to a sudden variation in wind speed from

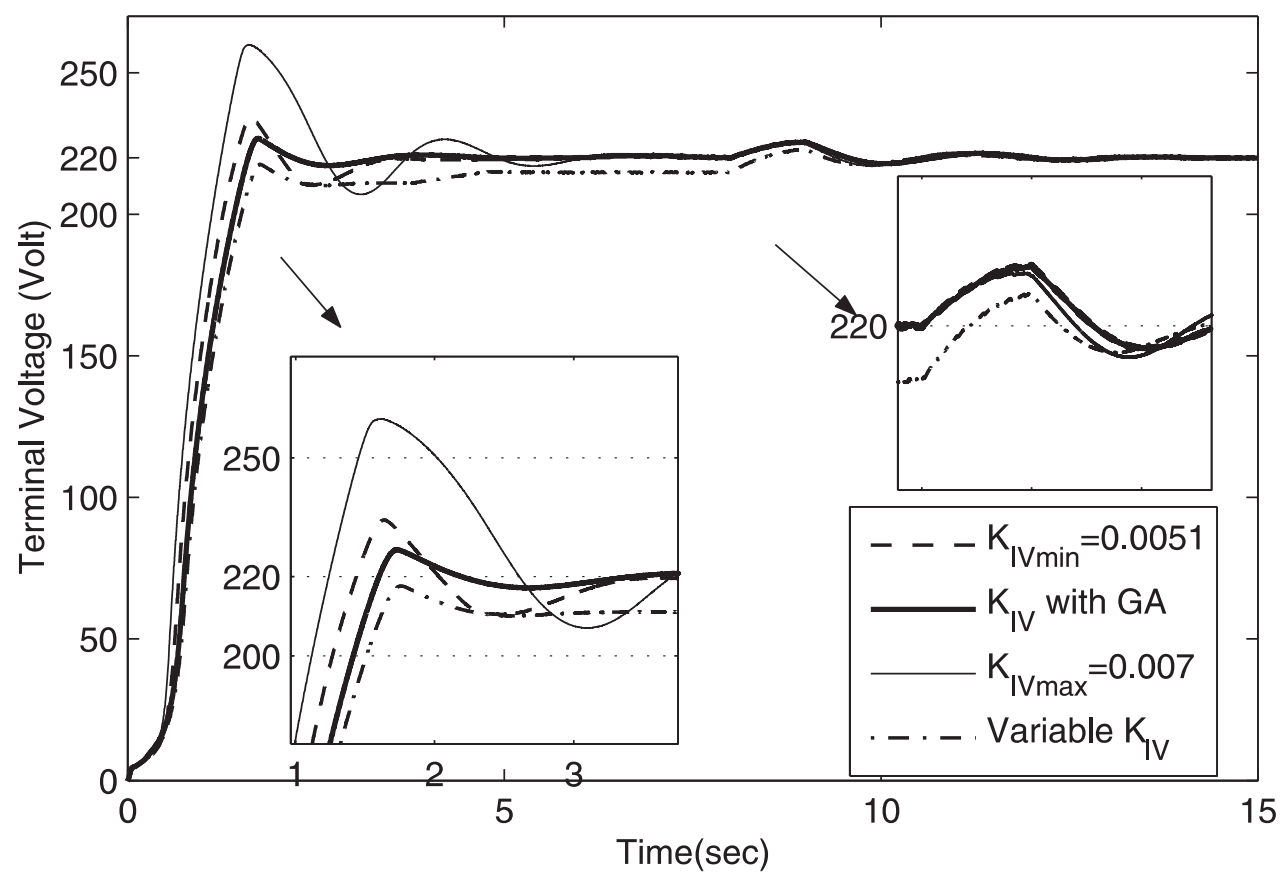

Fig. 14: Terminal voltage for PI and PI \& GA controllers 


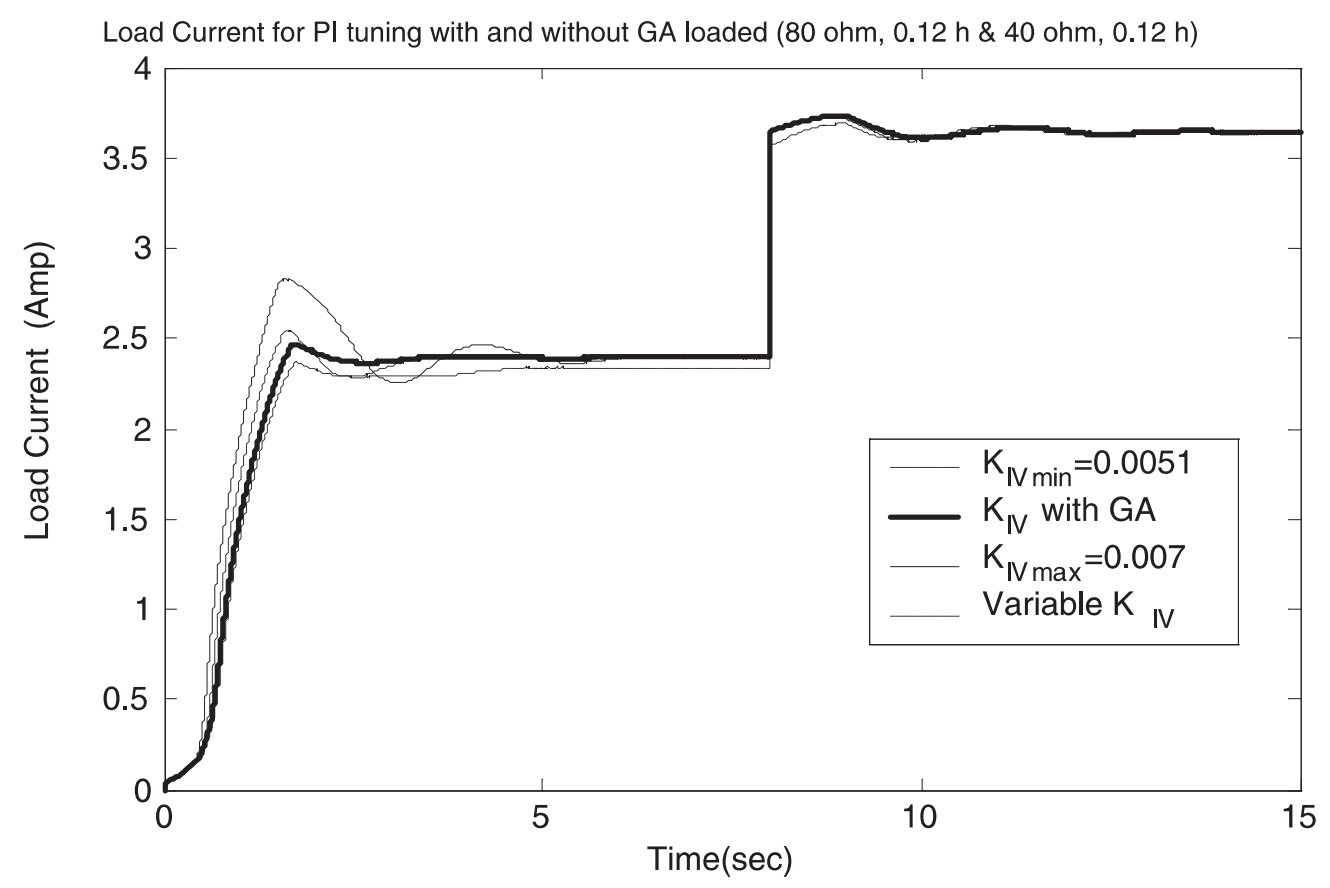

Fig. 15: Load current for PI and PI \& GA controllers

$7 \mathrm{~m} / \mathrm{s}$ to $15 \mathrm{~m} / \mathrm{s}$. Figs. 17,18 show the simulation results of the wind speed variation and the stator frequency, respectively.

The simulation result given in Fig. 18 shows the ability of the proposed controller to overcome the speed variation when using the variable and fixed integral gain.

\section{Conclusions}

This paper presents the application of two types of controller to enhance the performance of SEIG driven by WECS, using a variable rule based integral gain and GA. GA is used

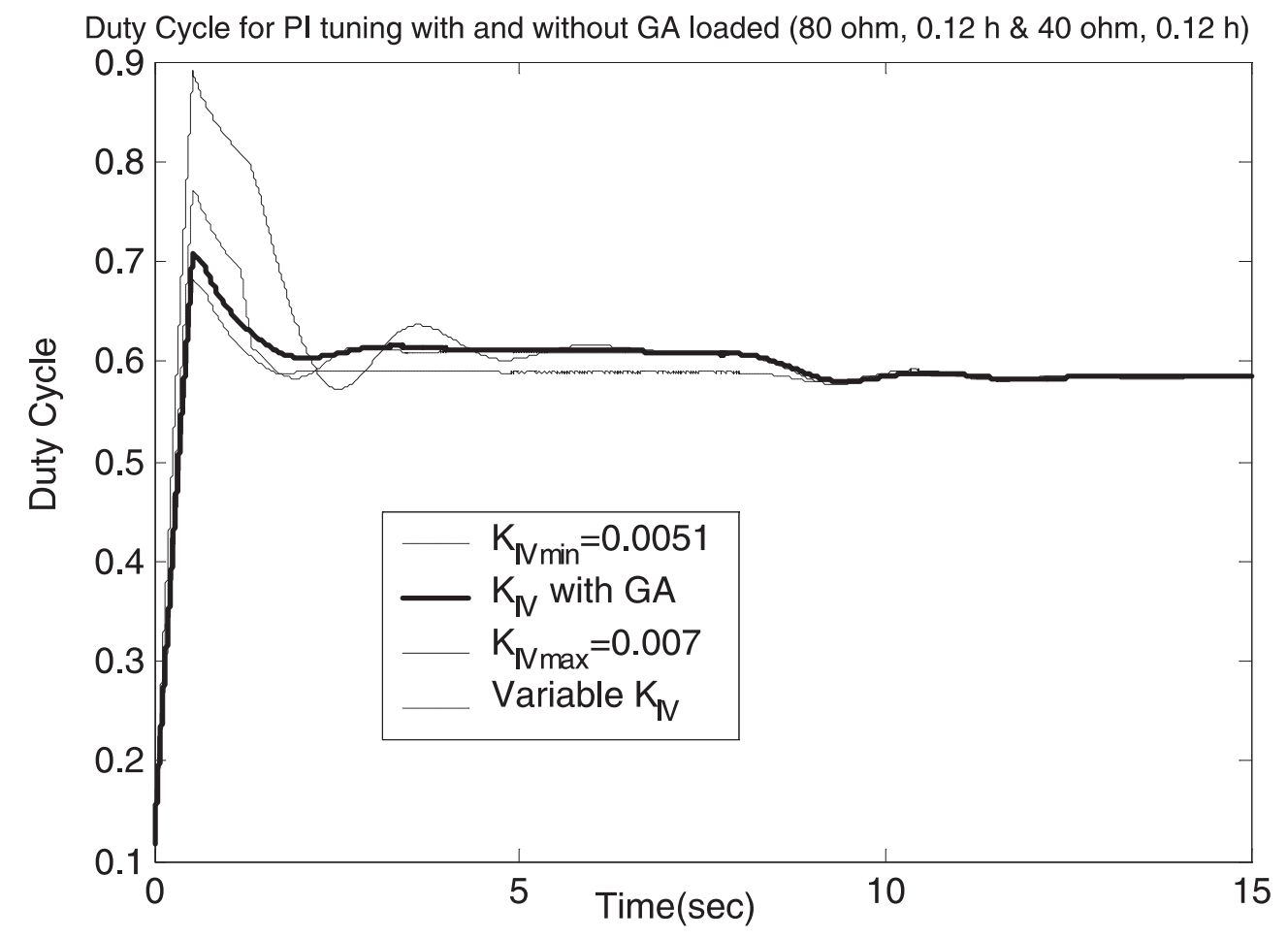

Fig. 16: Duty cycle for PI and PI \& GA controllers 


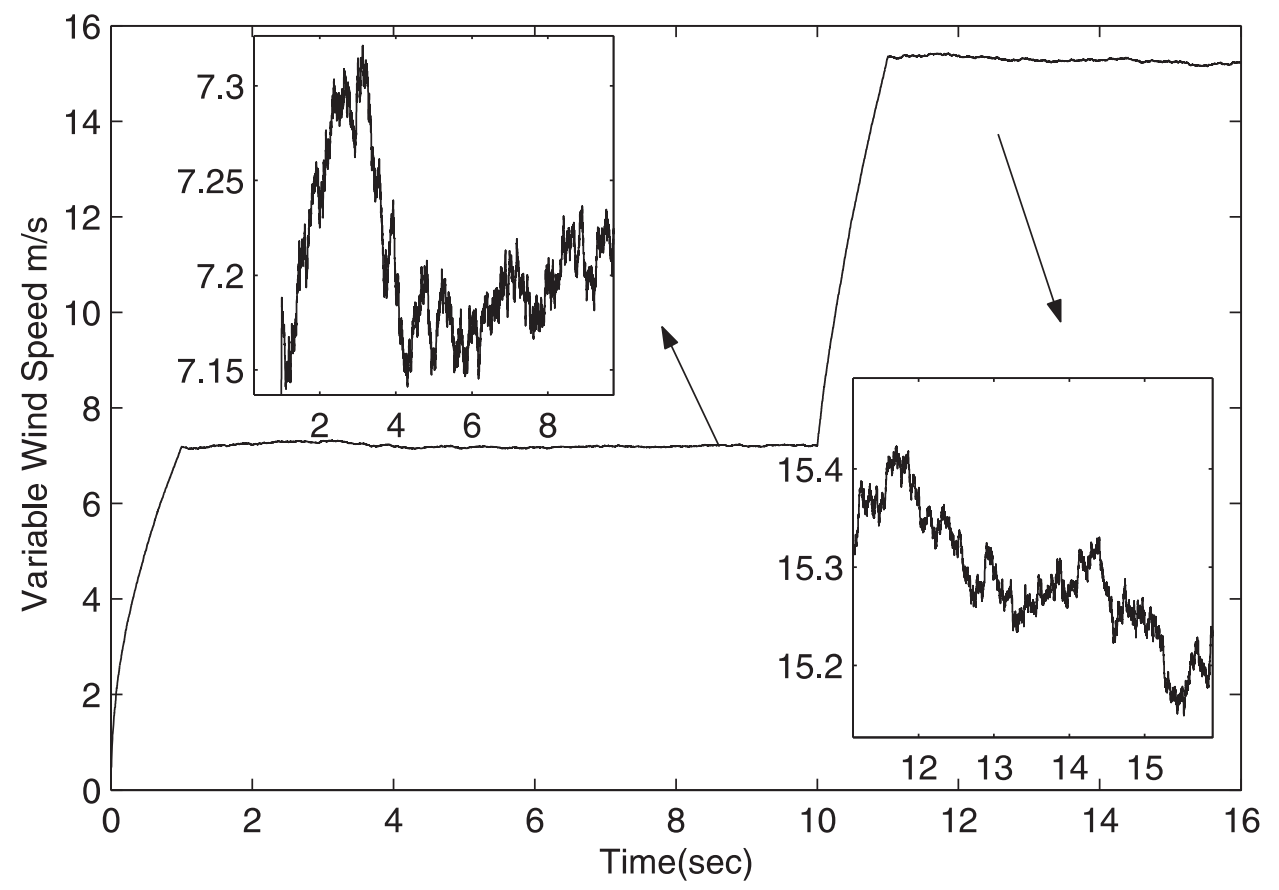

Fig. 17: Sudden variation for wind speed versus time

to optimize the PI controller gains in order to improve the dynamic response of the overall system. Optimal gains for the PI controller were determined using the GA procedure. The simulation results show that a PI controller tuned by the proposed GA was able to decrease the overshoot, and at same time to decrease the rising time. The simulation results show the effectiveness of the GA approach as a promising identification technique for PI controller gains. The two different

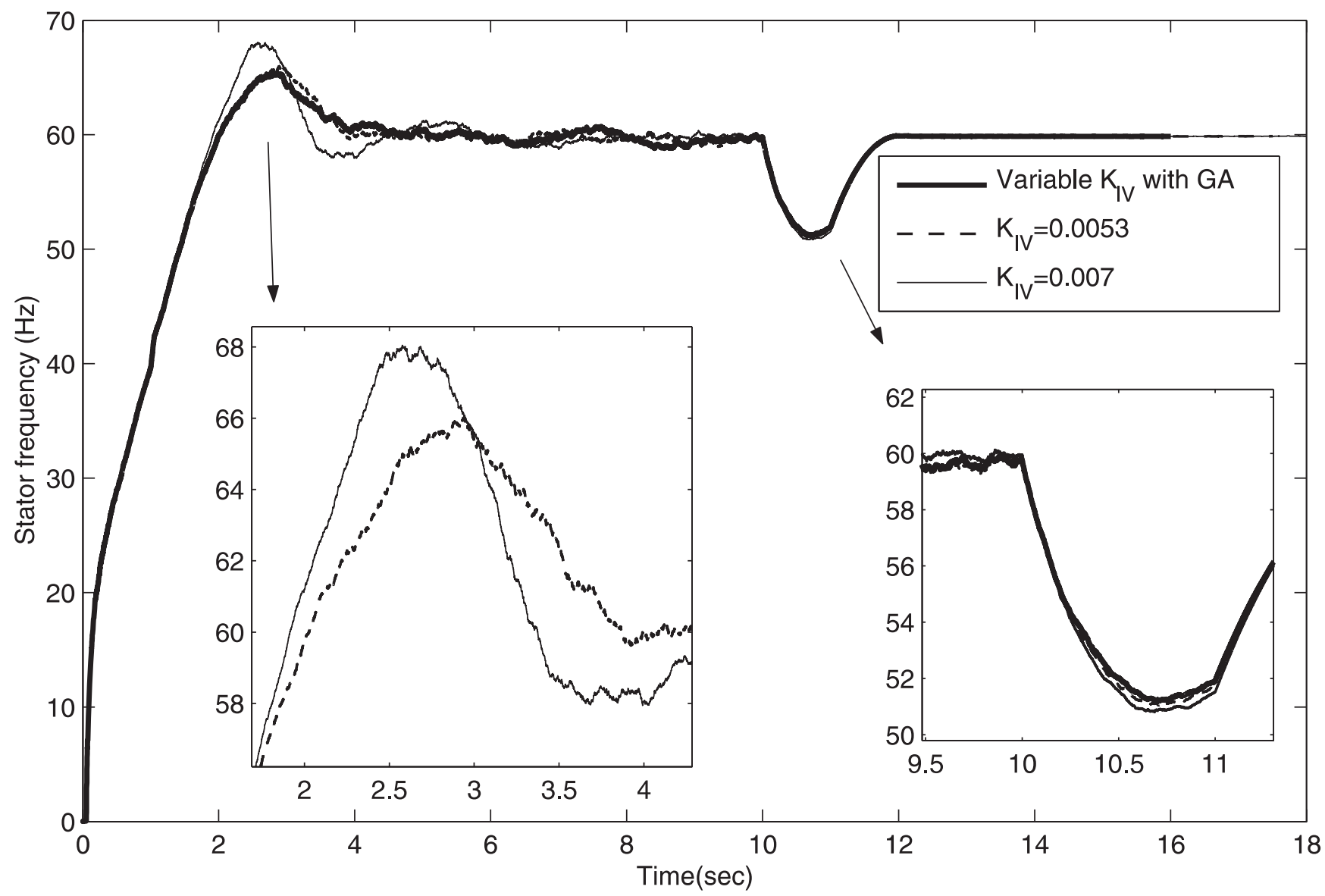

Fig. 18: Stator frequency according to wind speed variation for SEIG controlled by PI-GA 
types of controllers improve the dynamic performance when tested against warious types of disturbances.

\section{Appendix A - SEIG differential equation}

$V_{d s}$ (volt) Stator Voltage‘s Differential Equation at Direct Axis

$V_{d s}=-R_{s} \cdot i_{d s}-\left(\frac{\omega}{\omega_{b}}\right) \varphi_{q s}+p\left(\frac{\varphi_{d s}}{\omega_{b}}\right)$

$V_{q s} \quad$ Stator Voltage's Differential Equation at Quadrate Axis

$V_{q s}=-R_{s} \cdot i_{q s}-\left(\frac{\omega}{\omega_{b}}\right) \varphi_{d s}+p\left(\frac{\varphi_{q s}}{\omega_{b}}\right)$

$V_{d r} \quad$ Rotor Voltage‘s Differential Equation at Direct Axis

$V_{d r}=R_{r} \cdot i_{d r}-\left(\frac{\omega-\omega_{r}}{\omega_{b}}\right) \varphi_{q r}+p\left(\frac{\varphi_{d r}}{\omega_{b}}\right)$

$V_{q r} \quad$ Rotor Voltage's Differential Equation at Quadrate Axis

$V_{q r}=R_{r} \cdot i_{q r}-\left(\frac{\omega-\omega_{r}}{\omega_{b}}\right) \varphi_{d r}+p\left(\frac{\varphi_{q r}}{\omega_{b}}\right)$

Flux linkage differential equation for stator and rotor components:

$\varphi_{d s}=-X_{\ell s} \cdot i_{d s}+x_{m}\left(i_{d r}-i_{d s}\right)$,

where:

$\varphi_{d s}$ (Weber) is the stator flux linkage at the direct axis,

$i_{d r}$ (amp) is the rotor current at the direct axis but

$i_{d s}(\mathrm{amp})$ is the stator current at direct axis,

$p \quad$ is the differentiation parameter $=\mathrm{d} / \mathrm{d} t$.

$\varphi_{q s}=-X_{\ell s} \cdot i_{q s}+x_{m}\left(i_{q r}-i_{q s}\right)$,

where:

$\varphi_{q s}$ is the stator flux linkage at the quadrant axis,

$i_{q r}$ is the rotor current at quadrant axis but

$i_{q s} \quad$ is the stator current at the quadrant axis.

$\varphi_{d r}=X_{\ell r} \cdot i_{d r}+x_{m}\left(i_{d r}-i_{d s}\right)$,

where:

$\varphi_{d r}$ is the rotor flux linkage at the direct axis.

$\varphi_{q r}=X_{\ell r} \cdot i_{d s}+x_{m}\left(i_{q r}-i_{q s}\right)$,

where:

$\varphi_{q r} \quad$ is the rotor flux linkage at quadrant axis.

$\frac{\mathrm{d} \varphi_{q s}}{\mathrm{~d} t}=\omega_{b} \cdot\left(V_{q s}+R_{s} i_{q s}-\varphi_{d s}\right)$,

where:

$\omega_{b} \quad$ is the base speed.

$P_{m}=\frac{1}{8}\left(\pi \rho C_{p} D^{2} V_{w}^{3}\right)$

$C_{p}=\left[(0.44-0.0167 \beta) \cdot \sin \left(\frac{\pi(\mu-3)}{15-0.3 \beta}\right)-0.00184(\mu-3) \beta\right]$

Where:

$\omega_{m}(\mathrm{rad} / \mathrm{sec})$ is the mechanical speed
$P_{m}(\mathrm{~kW}) \quad$ is the mechanical power

$T_{m}(\mathrm{~nm}) \quad$ is the mechanical torque

$n$ (rpm) is the rotor revolution per minute

$C_{p} \quad$ is the power coefficient of the wind turbine

$\beta \quad$ is the blade pitch angle (degree)

$\mu \quad$ is the tip speed ratio

$V_{w}(\mathrm{~m} / \mathrm{s}) \quad$ is the wind speed

$D(\mathrm{~m}) \quad$ is the of the rotor Diameter of the wind turbine

$\pi$

$\rho\left(\mathrm{kg} / \mathrm{m}^{3}\right) \quad$ Air density

\section{Appendix B - SEIG parameters}

The induction machine under study as a SEIG has the following parameters:

$1.1 \mathrm{~kW}, 127 / 220 \mathrm{~V}$ (line voltage), 8.3/4.8 A (line current), $60 \mathrm{~Hz}, 2$ poles, wound-rotor induction machine [9, 10].

By choosing proper base values:

- base voltage $V_{b}=[220 /(1.73)] \mathrm{V}$,

- base current $I_{b}=4.8 \mathrm{~A}$,

- base impedance $Z_{b}=26.462 \mathrm{ohm}$,

- base rotor speed $N_{b}=3600 \mathrm{rpm}$, and

- base frequency $F_{b}=60 \mathrm{~Hz}$,

the per-unit parameters of the induction machine under study are equal:

- stator resistance $R_{s}=0.0779$,

- rotor resistance $R_{r}=0.0781$,

- stator reactance $X_{s}$ and rotor reactance $X_{r}$ are equal 0.0895 .

The equation of the motion of rotating parts of the combined studied SEIG and the wind turbine is also included in the system in order to provide a detailed simulation model. The inertia constant of the machine $H=0.055 \mathrm{~s}$.

\section{References}

[1] Li, Wang, Jian-Yi-Su: "Dynamic Performance of an Isolated Self Excited Induction Generator Under Various Loading Conditions", IEEE Transactions on Energy Conversion, Vol. 15 (1999), No. 1, March 1999, p. 93-100.

[2] Li, Wang, Ching- Huei Lee: "Long- Shunt and ShortShunt Connections on Dynamic Performance of a SEIG Feeding an Induction Motor Load", IEEE Transactions on Energy Conversion, Vol. 14 (2000), No. 1, p. 1-7.

[3] Golodberg, D.: Genetic Algorithms in Search Optimization and Machine Learning. Addision-Wesely, Reading, MA, 1989.

[4] Attia, A., Soliman, H.: "An Efficient Genetic Algorithm for Tuning PD Controller of Electric Drive for Astronomical Telescope." Scientific Bulletin of Ain Shams University, Faculty of Engineering, Part II, Issue No. 37/2, June 30, 2002.

[5] Ezzeldin, S. Abdin, Wilson, Xu: "Control Design and Dynamic Performance Analysis of a Wind Turbine Induction Generator Unit", IEEE Transaction on 
Energy Conversion, Vol. 15 (2000), No. 1, March 2000, p. 91-96.

[6] Marduchus, C.: "Switched Capacitor Circuits for Reactive Power Generation”, Ph.D. Thesis, Brunuel University, 1983.

[7] Sekaj, I.: "Genetic Algorithm - Based Control System Design and System Identification”, 5 ${ }^{\text {th }}$ International Mendel Conference on Soft Computing, 1999, Brno, Czech Republic, p. 139-144.

[8] Michalewic, Z.: Genetic Algorithms + Structure = Evolution Program. Springer-Verlag, Berlin Heidelberg, 1992.

Dr. Ing. Abdel-Fattah Attia phone:+202 5560046

fax:+2025548020

e-mail: attiaa1@yahoo.com

Astronomy Department
National Research Institute of Astronomy and Geophysics (NRIAG),

11421 Helwan

Cairo, EGYPT

Associate Prof. Dr. Ing. Hussein F. Soliman e-mail: hfaridsoliman@yahoo.com

Dept. of Electric Power and Machine Faculty of Engineering

Ain-Shams University

Abbasia, Cairo, EGYPT

Dr. Ing. Mokhymar Sabry

e-mail: sabry40@hotmail.com

Electricity \& Energy Ministry

New \& Renewable Energy Authority "NREA"

Wind Management, Cairo, EGYPT 